The Astrophysical Journal, 553:L63-L67, 2001 May 20

(C) 2001. The American Astronomical Society. All rights reserved. Printed in U.S.A.

\title{
HIGHLY ABUNDANT HCN IN THE INNER HOT ENVELOPE OF GL 2591: PROBING THE BIRTH OF A HOT CORE?
}

\author{
A. M. S. Boonman, ${ }^{1}$ R. Stark, ${ }^{2}$ F. F. S. van der Tak, ${ }^{2}$ E. F. van Dishoeck, ${ }^{1}$ P. B. van der Wal, ${ }^{2}$ \\ F. SCHÄFer, ${ }^{2}$ G. DE LANGe, ${ }^{3}$ AND W. M. LAAUWEN ${ }^{3}$ \\ Received 2000 December 20; accepted 2001 April 23; published 2001 May 8
}

\begin{abstract}
We present observations of the $\nu_{2}=0$ and vibrationally excited $\nu_{2}=1 J=9-8$ rotational lines of $\mathrm{HCN}$ at $797 \mathrm{GHz}$ toward the deeply embedded massive young stellar object GL 2591, which provide the missing link between the extended envelope traced by lower $J$ line emission and the small region of hot $\left(T_{\mathrm{ex}} \geq 300 \mathrm{~K}\right)$, abundant $\mathrm{HCN}$ seen in $14 \mu \mathrm{m}$ absorption with the Infrared Space Observatory (ISO). The line ratio yields $T_{\mathrm{ex}}=720_{-100}^{+135} \mathrm{~K}$, and the line profiles reveal that the hot gas seen with ISO is at the velocity of the protostar, arguing against a location in the outflow or in shocks. Radiative transfer calculations using a depth-dependent density and temperature structure show that the data rule out a constant abundance throughout the envelope but that a model with a jump of the abundance in the inner part by 2 orders of magnitude matches the observations. Such a jump is consistent with the sharp increase in $\mathrm{HCN}$ abundance at temperatures $\gtrsim 230 \mathrm{~K}$ predicted by recent chemical models in which atomic oxygen is driven into water at these temperatures. Together with the evidence for ice evaporation in this source, this result suggests that we may be witnessing the birth of a hot core. Thus, GL 2591 may represent a rare class of objects at an evolutionary stage just preceding the "hot core" stage of massive star formation.
\end{abstract}

Subject headings: circumstellar matter — ISM: abundances — ISM: individual (GL 2591) —

ISM: molecules — line: profiles — radiative transfer

\section{INTRODUCTION}

Molecules are important tools to investigate the physical and chemical structure of the envelopes around massive young stars. Physical models are a prerequisite for determining molecular abundance profiles, which in turn are powerful evolutionary indicators (van Dishoeck \& Blake 1998). HCN is a particularly important molecule because it has pure rotational lines in the submillimeter and rovibrational lines in the infrared part of the spectrum, which can both be observed from the ground. The combination of submillimeter emission and infrared absorption gives strong constraints on the structure and geometry of the sources (Carr et al. 1995; van der Tak et al. 1999, 2000). It also provides independent information on the level populations and thus allows a study of the excitation mechanisms present in these objects. $\mathrm{HCN}$ is one of the more abundant nitrogen-bearing species in dense clouds and plays a key role in the nitrogen chemistry in hot cores (Viti \& Williams 1999; Rodgers \& Charnley 2001). Therefore, it might also be a good candidate to probe the onset of the "hot core" phase in massive star-forming regions. This is the phase just before the ultracompact $\mathrm{H}$ II region is formed, in which the massive embedded young star still has a high accretion rate but begins to ionize its surrounding material, with thermal pressure creating a warm dense neutral shell. The hot core phase is characterized by weak radio continuum emission, evaporation of ices, and high-temperature chemistry ( $T \geqslant 300 \mathrm{~K}$ ) leading to enhanced gas-phase molecular abundances with respect to earlier evolutionary phases and the production of complex molecules, such as $\mathrm{CH}_{3} \mathrm{OCH}_{3}$ and $\mathrm{CH}_{3} \mathrm{CN}$ (Kurtz et al. 2000).

$\mathrm{HCN}$ has been detected toward many massive star-forming regions in the submillimeter and the infrared (e.g., Ziurys \& Turner 1986; Evans, Lacy, \& Carr 1991; Carr et al. 1995).

\footnotetext{
${ }^{1}$ Sterrewacht Leiden, P.O. Box 9513, 2300 RA Leiden, The Netherlands.

${ }^{2}$ Max-Planck-Institut für Radioastronomie, Auf dem Hügel 69, D-53121 Bonn, Germany.

${ }^{3}$ Space Research Organisation of the Netherlands (SRON), P.O. Box 800, 9700 AV Groningen, The Netherlands.
}

Typical HCN abundances toward massive protostars that do not show the presence of a hot core are $\sim 10^{-9}$ to $10^{-8}$, derived from submillimeter observations (Schreyer et al. 1997). Even most hot cores, which are predicted to have enhanced gasphase $\mathrm{HCN}$ abundances, show typical $\mathrm{HCN}$ abundances of $\sim 10^{-9}$ to $10^{-8}$, based on low- $J$ submillimeter lines (Hatchell, Millar, \& Rodgers 1998). On the other hand, observations with the Infrared Space Observatory (ISO) of the $\nu_{2}$ rovibrational band around $14 \mu \mathrm{m}$ toward a dozen massive young stars thought to precede the "hot core" phase have revealed strong HCN absorption indicating that hot gas $\left(T_{\mathrm{ex}}>300 \mathrm{~K}\right)$ is present with abundances up to $10^{-6}$ (Lahuis \& van Dishoeck 2000). Such high HCN abundances have been derived in very few massive star-forming regions from millimeter data, and, except for Orion, the high estimates are mostly based on interferometric observations of a single low- $J$ isotopic transition, leading to large uncertainties in the derived $\mathrm{HCN}$ abundances (Carral \& Welch 1992). Most of the well-known hot cores such as W3 $\left(\mathrm{H}_{2} \mathrm{O}\right)$ and $\mathrm{G} 34.3$, which show a wealth of complex organic molecules, are too weak at mid-infrared wavelengths to observe through infrared absorption with current instrumentation. The high-frequency submillimeter data presented here provide an alternative method to probe abundant $\mathrm{HCN}$.

The low spectral resolution of the ISO data $(R=\lambda / \Delta \lambda \sim$ 1500 at $14 \mu \mathrm{m}$ ) allows no kinematical information to be derived for the hot abundant $\mathrm{HCN}$ gas, and therefore its origin is not clear, in particular whether it is located in the inner hot part of the envelope or produced in shocks. Most of the sources showing hot $\mathrm{HCN}$ gas also possess significant outflows, so that shock chemistry cannot be ruled out. This is especially the case for the massive protostar GL 2591, where a significant outflow component is seen in infrared $\mathrm{CO}$ absorption lines (Mitchell et al. 1989; van der Tak et al. 1999). High-resolution heterodyne spectroscopy of high- $J$ rotational lines can distinguish between these two explanations. We present here observations of the $\mathrm{HCN}$ $\nu_{2}=0 J=9-8$ line and the first detection of the vibrationally excited $\nu_{2}=1 J=9-8$ line of $\mathrm{HCN}$ at $797.330 \mathrm{GHz}$ toward the massive protostar GL 2591. So far, the highest observed 
rotational transitions of $\mathrm{HCN}$ in this source are the $\nu_{2}=0$ $J=4-3$ and the vibrationally excited $\nu_{2}=1 J=4-3$ line (van der Tak et al. 1999). The advantage of the $J=9-8$ transitions is that they have higher excitation energies and can be observed at higher angular resolution than the low- $J$ transitions. In this way, the warmer inner part of the molecular envelope is uniquely probed. Using the low- $J \mathrm{HCN}, \mathrm{H}^{13} \mathrm{CN}$, and $\mathrm{HC}^{15} \mathrm{~N}$ rotational lines of van der Tak et al. (1999), only an HCN abundance for the outer envelope of GL 2591 could be derived. The addition of the $J=9-8$ lines allows us for the first time to construct an $\mathrm{HCN}$ abundance profile for both the inner and outer envelope. This will help to determine the evolutionary state of this massive protostar, especially whether it has already started the formation of a hot core.

\section{OBSERVATIONS}

The observations of the HCN $\nu_{2}=0$ and $\nu_{2}=1 J=9-8$ lines were made with the MPIfR/SRON $800 \mathrm{GHz}$ heterodyne spectrometer at the James Clerk Maxwell Telescope (JCMT) ${ }^{4}$ on 2000 April 19. The spectrometer is based on the MPIfR 795$880 \mathrm{GHz}$ quasi-optical SIS receiver employing standard niobium junction technology with planar tuning circuits fabricated from normal conducting aluminum. The receiver uses an InP Gunn oscillator followed by a doubler and a tripler stage. Details of the setup are described in Schäfer et al. (1997a, 1997b). For the measurements described here, a prototype waveguide mixer with a diagonal horn was used, which consists of a fixed-tuned waveguide mixer with an $\mathrm{Nb}$ SIS junction with $\mathrm{NbTiN}$ and $\mathrm{Al}$ wiring layers. These devices were fabricated at the University of Groningen. Details on the fabrication of similar SIS devices can be found in Jackson et al. (2001). This resulted in a double-sideband receiver noise temperature of about $550 \mathrm{~K}$ within a band of $50 \mathrm{GHz}$ centered at $810 \mathrm{GHz}$. The receiver has an intermediate frequency of $3.5 \mathrm{GHz}$ and a bandwidth of $1 \mathrm{GHz}$. Details of the receiver will be described elsewhere (R. Stark et al. 2001, in preparation). The double-sideband observations were taken under dry weather conditions yielding single-sideband system temperatures of about $6000 \mathrm{~K}$. The beam size at this frequency is about $8^{\prime \prime} \mathrm{FWHM}$, and the main-beam efficiency is $\eta_{\mathrm{MB}} \sim 0.2$. The absolute calibration uncertainty is estimated at $50 \%$. The digital autocorrelator spectrometer back end of the JCMT was used with a bandwidth of $500 \mathrm{MHz}$, yielding a spectral resolution of $378 \mathrm{kHz}\left(\sim 0.14 \mathrm{~km} \mathrm{~s}^{-1}\right)$. The observed spectrum of the massive protostar GL $2591\left[\alpha(1950)=20^{\mathrm{h}} 27^{\mathrm{m}} 35^{\mathrm{s}} .93, \delta(1950)=\right.$ $+40^{\circ} 01^{\prime} 14^{\prime \prime} .9$ ], taken by beam switching over $180^{\prime \prime}$, is shown in Figure 1, and the integrated line intensities are listed in Table 1.

\section{RESULTS}

Two lines are detected in the spectrum of GL 2591 (Fig. 1). The strongest is identified as the $J=9-8$ rotational transition of $\mathrm{HCN}$ in the vibrational ground state $\nu_{2}=0$. This is the first detection of this line outside Orion (Stutzki et al. 1988). The other line is the first detection of the vibrationally excited $\nu_{2}=1 J=9-8$ line of $\mathrm{HCN}$ at $797.330 \mathrm{GHz}$ in a massive protostar. This line arises from $\sim 1200 \mathrm{~K}$ above the vibrational ground state and is therefore an even better probe of the warm gas in this protostar than the $\nu_{2}=0 J=9-8$ transition, which has an excitation energy of $\sim 190 \mathrm{~K}$. Both lines are resolved and can be fitted by a single Gaussian. The width of the

\footnotetext{
${ }^{4}$ The James Clerk Maxwell Telescope is operated by the Joint Astronomy Centre, on behalf of the Particle Physics and Astronomy Research Council of the United Kingdom, the Netherlands Organization for Scientific Research, and the National Research Council of Canada.
}
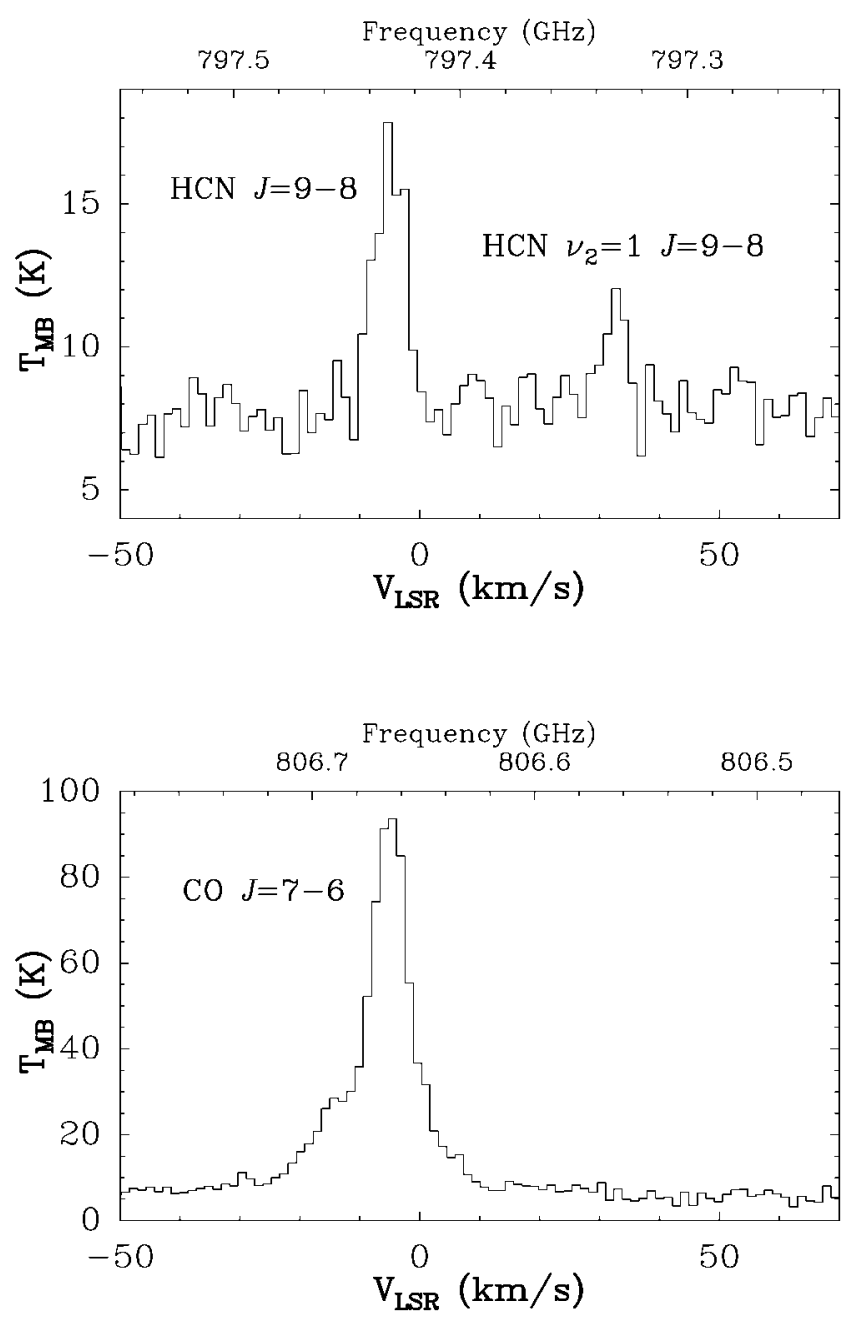

FIG. 1.-Top: Spectrum of the $\nu_{2}=0 J=9-8$ and the vibrationally excited $\nu_{2}=1 J=9-8$ line of $\mathrm{HCN}$ at 797.434 and $797.330 \mathrm{GHz}$, respectively, toward the massive protostar GL 2591. The spectrum has been smoothed to a spectral resolution of $\sim 1.4 \mathrm{~km} \mathrm{~s}^{-1}$. Bottom: The CO $J=7-6$ line at $806.652 \mathrm{GHz}$ is shown for comparison. The latter spectrum shows clearly the presence of a wing component, whereas the HCN $J=9-8$ lines do not show such a component. The vertical offset shows the submillimeter continuum due to warm dust, which agrees within $30 \%$ with the $350 \mu \mathrm{m}$ continuum photometry of this source (van der Tak et al. 2000).

$\nu_{2}=0 \mathrm{~J}=9-8$ line is $5.8 \pm 0.4 \mathrm{~km} \mathrm{~s}^{-1}$ and of the $\nu_{2}=1$ line is $4.4 \pm 0.4 \mathrm{~km} \mathrm{~s}^{-1}$. The position of the line center of $V_{\mathrm{LSR}}=-5.1 \pm 0.5 \mathrm{~km} \mathrm{~s}^{-1}$ for the $\nu_{2}=0$ line and of $V_{\mathrm{LSR}}=-6.1 \pm 0.5 \mathrm{~km} \mathrm{~s}^{-1}$ for the $\nu_{2}=1$ line is consistent with the velocity of the quiescent envelope gas of $V_{\mathrm{LSR}}=$ $-5.5 \pm 0.2 \mathrm{~km} \mathrm{~s}^{-1}$ (van der Tak et al. 1999). Although our observation of the CO $J=7-6$ line in the same beam clearly shows the presence of an outflow component (Fig. 1), the HCN lines have no wings at a level larger than $\sim 17 \%$ of the peak intensity, much lower than the $\sim 30 \%$ level of the CO $J=$ 7-6 wings. This suggests that both HCN $J=9-8$ lines originate in the warm quiescent gas of the molecular envelope. Moreover, since the vibrationally excited $\nu_{2}=1 J=9-8$ and the ground-state $\nu_{2}=0 J=9-8 \mathrm{HCN}$ lines are observed in the same spectrum and in the same sideband, their relative calibration is better than $25 \%$. Also, modeling shows that both lines are optically thin (Table 1), so that their relative strength provides a very accurate handle on the vibrational temperature. We derive $T_{\mathrm{ex}}=720_{-100}^{+135} \mathrm{~K}$, in excellent agreement with the value $T_{\text {ex }}=600_{-50}^{+75} \mathrm{~K}$, inferred from the mid-infrared rovibra- 
TABLE 1

Comparison of Observed Integrated Intensities with Different Model Results

\begin{tabular}{|c|c|c|c|c|c|c|c|}
\hline Species & Band & Transition & $\begin{array}{c}\text { Model 1 } \\
\left(\mathrm{K} \mathrm{km} \mathrm{s}^{-1}\right)\end{array}$ & $\begin{array}{c}\text { Model } 2 \\
\left(\mathrm{~K} \mathrm{~km} \mathrm{~s}^{-1}\right)\end{array}$ & $\begin{array}{c}\text { Model } 3 \\
\left(\mathrm{~K} \mathrm{~km} \mathrm{~s}^{-1}\right)\end{array}$ & $\begin{array}{c}\text { Observed } \\
\left(\mathrm{K} \mathrm{km} \mathrm{s}^{-1}\right)\end{array}$ & Optical Depth ${ }^{\mathrm{a}}$ \\
\hline \multirow[t]{4}{*}{$\mathrm{HCN}$} & $\nu_{2}=0$ & $J=4-3$ & 19.38 & 20.09 & 22.95 & $24.7^{\mathrm{b}}$ & 9.9 \\
\hline & $\nu_{2}=0$ & $J=9-8$ & 14.64 & 32.52 & 57.51 & 60.2 & 0.14 \\
\hline & $\nu_{2}=1$ & $J=4-3$ & 0.11 & 0.44 & 2.87 & 2.5 & 0.006 \\
\hline & $\nu_{2}=1$ & $J=9-8$ & 0.39 & 3.25 & 22.10 & 17.1 & 0.03 \\
\hline \multirow[t]{2}{*}{$\mathrm{H}^{13} \mathrm{CN}$} & $\nu_{2}=0$ & $J=3-2$ & 1.93 & $\ldots$ & 3.70 & 5.5 & 0.33 \\
\hline & $\nu_{2}=0$ & $J=4-3$ & 1.05 & $\ldots$ & 4.72 & 8.6 & 0.11 \\
\hline \multirow[t]{2}{*}{$\mathrm{HC}^{15} \mathrm{~N}$} & $\nu_{2}=0$ & $J=3-2$ & 0.45 & $\ldots$ & 1.03 & 2.4 & 0.07 \\
\hline & $\nu_{2}=0$ & $J=4-3$ & 0.24 & $\ldots$ & 1.41 & 3.1 & 0.03 \\
\hline$N(\mathrm{HCN})\left(\times 10^{16} \mathrm{~cm}^{-2}\right)$ & & & 0.12 & 0.36 & 2.7 & & \\
\hline
\end{tabular}

Note.-Model 1: constant abundance $x(\mathrm{HCN})=10^{-8}$. Model 2: $x(\mathrm{HCN})=10^{-8}$ for $T<230 \mathrm{~K}, x(\mathrm{HCN})=10^{-7}$ for $T>230 \mathrm{~K}$. Model 3: $x(\mathrm{HCN})=10^{-8}$ for $T<230 \mathrm{~K}, x(\mathrm{HCN})=10^{-6}$ for $T>230 \mathrm{~K}$. The model $\mathrm{H}^{13} \mathrm{CN}$ and $\mathrm{HC}^{15} \mathrm{~N}$ abundances correspond to the $\mathrm{HCN}$ abundance divided by the isotopic ratios ${ }^{12} \mathrm{C} /{ }^{13} \mathrm{C}=60$ and ${ }^{14} \mathrm{~N} /{ }^{15} \mathrm{~N}=270$, respectively (Wilson \& Rood 1994). Calibration uncertainties in the observed integrated intensities are $\sim 50 \%$ for the $J=9-8$ lines and $\sim 30 \%$ for the other lines (van der Tak et al. 1999).

${ }^{a}$ The optical depth corresponding to model 3.

${ }^{\mathrm{b}}$ The narrow component at $V_{\mathrm{LSR}}=-5.7 \mathrm{~km} \mathrm{~s}^{-1}$ (van der Tak et al. 1999).

tional absorption band observed with ISO (Lahuis \& van Dishoeck 2000). This strengthens our main argument that the HCN $J=9-8$ lines and the rovibrational absorption band seen with $I S O$ probe the same hot region. The continuum present in the spectra is due to warm dust, and its level agrees within $30 \%$ with the $350 \mu \mathrm{m}$ continuum photometry of this source (van der Tak et al. 2000).

\section{MODELS AND ANALYSIS}

The data were analyzed using the temperature and density gradients derived for GL 2591 by van der Tak et al. (2000), shown in Figure 2. This model is based on submillimeter dust continuum and $\mathrm{CS}$ and $\mathrm{H}_{2} \mathrm{CO}$ line emission data and also reproduces infrared absorption measurements of $\mathrm{CO}$. The radiative transfer and excitation of $\mathrm{HCN}$ was calculated with the Monte Carlo code of Hogerheijde \& van der Tak (2000) on a grid of 40 concentric shells, assuming spherical geometry. The calculations include energy levels up to $J=21$ in both the $\nu_{2}=0$ and $\nu_{2}=1$ states and use collisional rate coefficients by S. Green. ${ }^{5}$ For transitions between vibrational levels, a rate coefficient of $10^{-12} \mathrm{~cm}^{3} \mathrm{~s}^{-1}$ was assumed. Radiative excitation

${ }^{5}$ See http://www.giss.nasa.gov/data/mcrates.

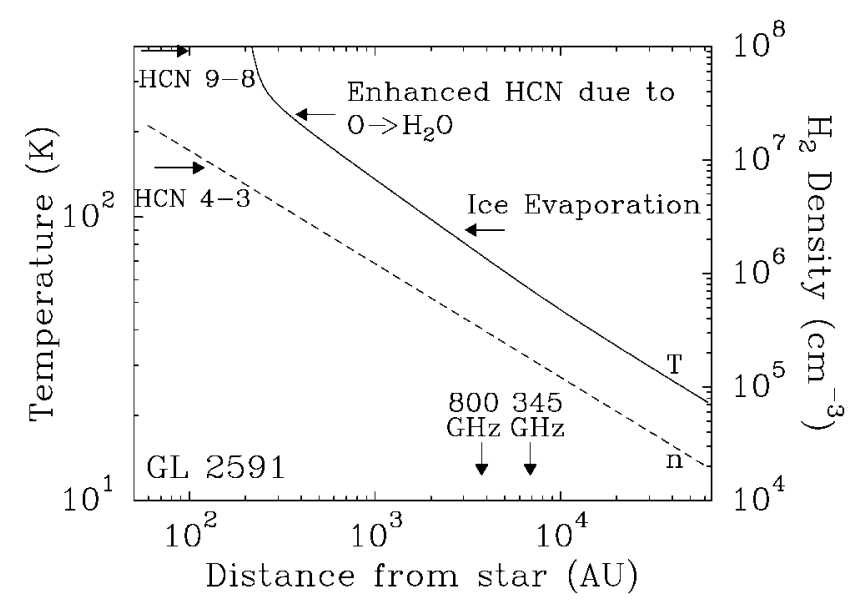

FIG. 2.-Temperature and density structure of GL 2591. The critical densities for the $\nu_{2}=0 J=9-8$ and $\nu_{2}=0 J=4-3$ lines are indicated as well as the JCMT beam sizes at the different frequencies (adapted from van Dishoeck \& van der Tak 2000). through the $14 \mu \mathrm{m}$ band due to warm dust mixed with the gas was also included, using grain opacities from Ossenkopf \& Henning (1994) and assuming $T_{\text {dust }}=T_{\text {gas }}$. No external radiation field apart from the $2.73 \mathrm{~K}$ cosmic background radiation was applied. Comparison with the observed emission proceeds by convolving the calculated sky brightness with the appropriate beam pattern.

In addition to the $\mathrm{HCN} J=9-8$ lines discussed here, the low$J$ HCN and isotopic lines from van der Tak et al. (1999) (see Table 1) are used for comparison with the model. It was found that this model cannot reproduce the new data using the $\mathrm{HCN}$ abundances $x(\mathrm{HCN})=n(\mathrm{HCN}) / n\left(\mathrm{H}_{2}\right)$ of a few times $10^{-8}$ indicated by the low- $J$ isotopic lines. This point is illustrated by model 1 in Table 1 . However, using the abundance of $\sim 10^{-6}$ indicated by the $\nu_{2}=1$ lines and the ISO observations overproduces the low- $J$ line data by an order of magnitude. Therefore, a model has been used with different $\mathrm{HCN}$ abundances in the inner and outer parts of the circumstellar envelope (Fig. 3). The "jump" is applied at $\approx 350 \mathrm{AU}$ from the star where $T=230 \mathrm{~K}$. Chemical models predict that at this gas temperature, reactions of $\mathrm{O}$ and $\mathrm{OH}$ with $\mathrm{H}_{2}$ drive atomic oxygen into water (Charnley 1997; Rodgers \& Charnley 2001). Interferometric observations of $\mathrm{HCN} J=1-0$ already indicated that the $\mathrm{HCN}$ enhancement

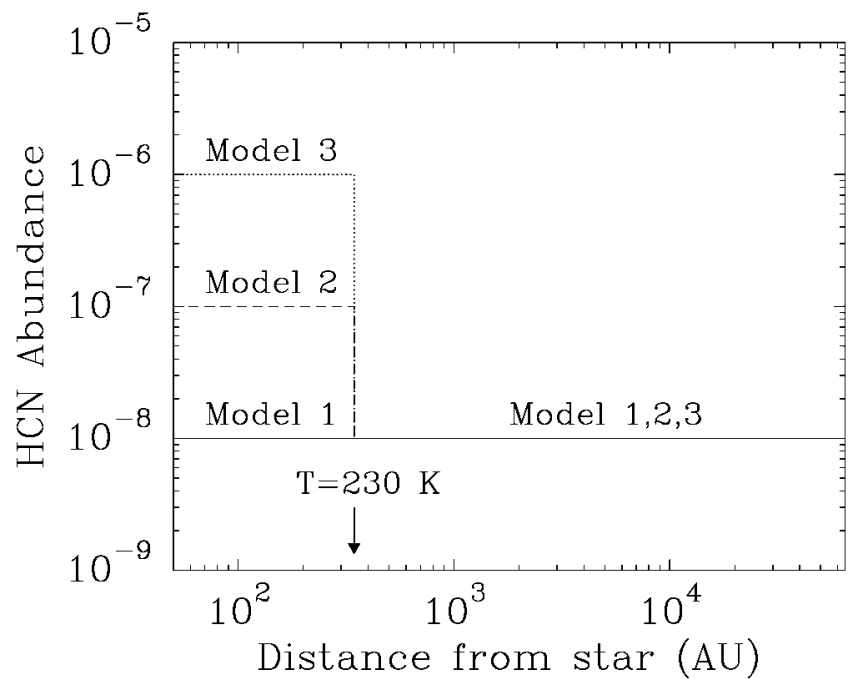

FIG. 3.-Different $\mathrm{HCN}$ abundance profiles used in the analysis of $\S 4$ (see also Table 1). 
must be limited to a region smaller than 1500 AU where $T>$ $120 \mathrm{~K}$ and is therefore due to gas-phase reactions rather than ice evaporation at $T \sim 90 \mathrm{~K}$ (van der Tak et al. 1999).

Increasing the $\mathrm{HCN}$ abundance in the inner envelope by a factor of 10 (model 2) still underproduces the observed integrated intensities (Table 1). An HCN abundance of $x(\mathrm{HCN})=10^{-6}$ in the inner envelope, combined with the outer envelope abundance of $x(\mathrm{HCN})=10^{-8}$ (model 3$)$, reproduces the observed values of the HCN lines within $\sim 10 \%$ (Table 1). The inner envelope $\mathrm{HCN}$ abundance $x(\mathrm{HCN})=10^{-6}$ corresponds well with the value $x(\mathrm{HCN})=(6.6 \pm 1.0) \times 10^{-7}$ derived for the hot gas from $I S O$ observations. The HCN column density derived from the latter model (Table 1) also matches best the value of $N(\mathrm{HCN})=(4 \pm 0.6) \times 10^{16} \mathrm{~cm}^{-2}$ derived from the same observations by Lahuis \& van Dishoeck (2000) and the value for the warm $(T \geq 200 \mathrm{~K})$ gas of $N(\mathrm{HCN}) \sim(3-4) \times 10^{16} \mathrm{~cm}^{-2}$ derived from ground-based observations by Carr et al. (1995). The $\mathrm{H}^{13} \mathrm{CN}$ and $\mathrm{HC}^{15} \mathrm{~N}$ lines are reproduced within a factor of about 2 .

Decreasing the inner radius of the model (Fig. 2) by a factor of 3 and extrapolating the temperature and density profile yields similar (within 10\%) results for model 3. The corresponding $\mathrm{HCN}$ column density of $N(\mathrm{HCN})=6.6 \times 10^{16} \mathrm{~cm}^{-2}$ is somewhat larger than that found from the infrared observations.

Our analysis shows that both $\nu_{2}=1$ lines and the $\nu_{2}=0$ $J=9-8$ line are very sensitive to different abundances in the inner envelope, contrary to the $\nu_{2}=0 \mathrm{~J}=4-3$ line, which is optically thick (Table 1) and traces only the outer envelope. The low- $J$ isotopic lines are also sensitive to different abundances in the inner envelope, suggesting that the warm gas contributes significantly to their observed line profiles. This is consistent with the maximum optical depths of $\leq 0.33$ derived for these lines (Table 1).

\section{DISCUSSION}

The HCN abundance of $\sim 10^{-6}$ found here for the inner ( $\$ 350 \mathrm{AU}$ ) hot envelope of GL 2591 is much higher than predicted by gas-phase models of cold dense clouds of $\sim 10^{-9}$ to $10^{-8}$ (Lee, Bettens, \& Herbst 1996; Millar, Farquhar, \& Willacy 1997; Herbst, Terzieva, \& Talbi 2000). Models for hightemperature regions predict, however, $\mathrm{HCN}$ abundances of up to $\sim 10^{-6}$ to $10^{-5}$ (Caselli, Hasegawa, \& Herbst 1993; Charnley 1997; Viti \& Williams 1999; Rodgers \& Charnley 2001). While the present data do not allow a precise determination of where the jump occurs, our finding is consistent with the recent chemical models by Charnley (1997) and Rodgers \& Charnley (2001), who show that above a critical temperature of 230-300 K, the reactions $\mathrm{O}+\mathrm{H}_{2} \rightarrow \mathrm{OH}+\mathrm{H}, \mathrm{OH}+\mathrm{H}_{2} \rightarrow \mathrm{H}_{2} \mathrm{O}+\mathrm{H}$ drive most of the atomic oxygen into $\mathrm{H}_{2} \mathrm{O}$. This results in low gas-phase $\mathrm{O}_{2}$ and enhanced atomic $\mathrm{C}$ abundances, since gas-phase $\mathrm{O}_{2}$ is one of the principal destroyers of atomic C. The formation of atomic $\mathrm{C}$ is through cosmic-ray-induced photodissociation of $\mathrm{CO}$ and thus is not changed. The formation of $\mathrm{N}_{2}$ through $\mathrm{NO}+\mathrm{N} \rightarrow \mathrm{N}_{2}+\mathrm{O}$ is also suppressed at high temperatures, since less NO is available. This provides more reactive atomic nitrogen in the gas phase. These enhanced atomic $\mathrm{C}$ and $\mathrm{N}$ abundances result in a significantly increased gas-phase $\mathrm{HCN}$ abundance at temperatures $\gtrsim 230-300 \mathrm{~K}$. Indeed, a recent chemical model by S. Doty (2001, private communication) using the temperature and density gradient of GL 2591 predicts such a sharply increasing HCN abundance profile. The prediction by these models of a large fraction of oxygen in atomic form in the outer envelope and in water in the inner part is consistent with the nondetection of molecular oxygen by the Submillimeter Wave Astronomy Satellite in this region (E. Bergin 2000, private communication). $\mathrm{HCN}$ ice has not been detected yet, and, as discussed above, interferometric observations indicate that the HCN enhancement cannot be explained by ice evaporation alone. However, ice evaporation of other species such as $\mathrm{H}_{2} \mathrm{O}, \mathrm{CO}_{2}$, and $\mathrm{C}_{2} \mathrm{H}_{2}$ has occurred for GL 2591 (van Dishoeck 1998). Together with the evidence for high-temperature chemistry inferred for $\mathrm{HCN}$, this suggests that we may be witnessing the formation of a hot core in GL 2591. Thus, GL 2591 may represent a rare class of objects at an evolutionary stage just preceding the "hot core" stage of massive star formation.

Inclusion of a chemistry network in the modeling will allow a refinement of our abundance profile. Interferometric observations of high- $J$ HCN transitions at subarcsecond resolution are needed to determine the temperature at which the jump takes place more accurately. So far, such a high HCN abundance as derived for the inner part of the molecular envelope in GL 2591 has been observed in very few massive star-forming regions other than Orion. Therefore, observations of high- $J$ $\mathrm{HCN}$ vibrational ground-state and vibrationally excited $\nu_{2}=$ 1 rotational lines toward other massive protostars, including hot cores, will allow a comparison of abundance profiles between these sources and a search for evolutionary effects. Finally, high-resolution observations of other "hot core" molecules, such as $\mathrm{CH}_{3} \mathrm{OCH}_{3}$ in the submillimeter or $\mathrm{C}_{2} \mathrm{H}_{2}$ in the infrared, may confirm the presence of a hot core in GL 2591.

The support of Rolf Güsten, Ian Robson, and Paul Wesselius in bringing the MPIfR/SRON $800 \mathrm{GHz}$ heterodyne instrument to the JCMT is greatly appreciated. It is a pleasure to thank the JCMT staff and the MPIfR division for submillimeter technology for their outstanding support. This work was partly supported by NWO grant 614-41-003. E. F. v. D. is grateful to the Miller Research Institute and the Department of Astronomy at the University of California at Berkeley for their hospitality.

\section{REFERENCES}

Carr, J. S., Evans, N. J., II, Lacy, J. H., \& Zhou, S. 1995, ApJ, 450, 667

Carral, P., \& Welch, W. J. 1992, ApJ, 385, 244

Caselli, P., Hasegawa, T. I., \& Herbst, E. 1993, ApJ, 408, 548

Charnley, S. B. 1997, ApJ, 481, 396

Evans, N. J., II, Lacy, J. H., \& Carr, J. S. 1991, ApJ, 383, 674

Hatchell, J., Millar, T. J., \& Rodgers, S. D. 1998, A\&A, 332, 695

Herbst, E., Terzieva, R., \& Talbi, D. 2000, MNRAS, 311, 869

Hogerheijde, M. R., \& van der Tak, F. F. S. 2000, A\&A, 362, 697

Jackson, B. D., de Lange, G., Laauwen, W. M., Gao, J. R., Iosad, N. N., \& Klapwijk T. M. 2001, in Proc. 11th Int. Symp. on Space Terahertz Technology, in press
Kurtz, S., Cesaroni, R., Churchwell, E., Hofner, P., \& Walmsley, C. M. 2000, in Protostars and Planets IV, ed. V. Mannings, A. P. Boss, \& S. S. Russell (Tucson: Univ. Arizona Press), 299

Lahuis, F., \& van Dishoeck, E. F. 2000, A\&A, 355, 699

Lee, H.-H., Bettens, R. P. A., \& Herbst, E. 1996, A\&AS, 119, 111

Millar, T. J., Farquhar, P. R. A., \& Willacy, K. 1997, A\&AS, 121, 139

Mitchell, G. F., Curry, C., Maillard, J.-P., \& Allen, M. 1989, ApJ, 341, 1020

Ossenkopf, V., \& Henning, Th. 1994, A\&A, 291, 943

Rodgers, S. D., \& Charnley, S. B. 2001, ApJ, 546, 324

Schäfer, F., van der Wal, P., Kreysa, E., \& Gundlach, K. H. 1997a, Proc. Eighth Int. Symp. on Space Terahertz Technology, ed. R. Blundell \& E. Tong (Cambridge: HSCA), 566 
Schäfer, F., van der Wal, P., Kreysa, E., Lehnert, T., \& Gundlach, K. H. 1997b, in Proc. Fifth Int. Workshop on Terahertz Electronics, ed. K. H. Gundlach \& M. Carter (Grenoble: IRAM)

Schreyer, K., Helmich, F. P., van Dishoeck, E. F., \& Henning, Th. 1997, A\&A, 326, 347

Stutzki, J., Genzel, R., Harris, A. I., Herman, J., \& Jaffe, D. T. 1988, ApJ, 330, L125

van der Tak, F. F. S., van Dishoeck, E. F., Evans, N. J., II, Bakker, E. J., \& Blake, G. A. 1999, ApJ, 522, 991 van der Tak, F. F. S., van Dishoeck, E. F., Evans, N. J., II, \& Blake, G. A. 2000, ApJ, 537, 283

van Dishoeck, E. F. 1998, Faraday Discuss., 109, 31

van Dishoeck, E. F., \& Blake, G. A. 1998, ARA\&A, 36, 317

van Dishoeck, E. F., \& van der Tak, F. F. S. 2000, in IAU Symp. 197, Astrochemistry: From Molecular Clouds to Planetary Systems, ed. Y. C. Minh \& E. F. van Dishoeck (San Francisco: ASP), 97

Viti, S., \& Williams, D. A. 1999, MNRAS, 305, 755

Wilson, T. L., \& Rood, R. 1994, ARA\&A, 32, 191

Ziurys, L. M., \& Turner, B. E. 1986, ApJ, 300, L19 